Active and Passive Electronic Components, September 2004, Vol. 27, pp. 161-167

\title{
NOVEL TWO-DIMENSIONAL (2-D) DEFECTED GROUND ARRAY FOR PLANAR CIRCUITS
}

\author{
HAIWEN LIU ${ }^{\mathrm{a}, \mathrm{b}, *}$, XIAOWEI SUN ${ }^{\mathrm{b}}$ and ZHENGFAN LI $^{\mathrm{a}}$ \\ ${ }^{a}$ Department of Electronic Engineering, Shanghai Jiaotong University, P.O. Box 006, No. 1954, \\ Huashan Rd., Shanghai 200030, China; ${ }^{\mathrm{b}}$ Shanghai Institute of Microsystem and \\ Information Technology, Chinese Academy of Sciences, Shanghai 200050, China
}

(Received 3 July 2003; In final form 28 July 2003)

\begin{abstract}
This paper presents a novel two-dimensional (2-D) defected ground array (DGA) for planar circuits, which has horizontal and vertical periodicities of defect structure. The defected unit cell of DGA is composed of a Sierpinski carpet structure to improve the effective inductance. Measurements show that the proposed DGA provides steeper cutoff characteristics, lower cutoff frequency, and higher slow-wave factors than the conventional periodic defected ground structure in the same occupied surface.
\end{abstract}

Keywords: Defected ground array; Defected ground structure (DGS); Fractal; Cutoff frequency; Slow-wave factor

\section{INTRODUCTION}

The rapid development of three-dimensional microwave integrated circuits (MICs), microwave monolithic integrated circuits (MMICs), and multiplayer-oriented low-temperature cofired-ceramic (LTCC) fabrication and processing techniques in recent years has led to great interest being directed towards the design of high-quality planar circuits $[1,2]$.

Defected ground structure (DGS) for the microstrip line, which has etched defects in the backside metallic ground plane, is one recent hotspot of microwave circuit design $[3,4]$. Compared to photonic bandgap (PBG) [5], DGS has a simple structure and potentially great applicability to design microwave circuits. Two of the most important characteristics of DGS are bandgap and high slow-wave effects, which are caused by the equivalent L-C components [3]. The microstrip lines with DGS have much higher impedance and increased slow-wave factor than conventional lines. Thus, the planar circuits can be reduced greatly while their performances keep well. One-dimensional (1-D) periodic DGS have been reported and applied to amplifiers [6], oscillators [7] and coplanar waveguide [8].

In this letter, a novel two-dimensional (2-D) defected-ground array (DGA) for planar circuits is proposed. The defected unit cell of DGA is composed of a Sierpinski carpet structure

\footnotetext{
* Corresponding author. Tel.: (86-21) 62511070 ext. 8603; Fax: (86-21) 62933001; E-mails: haiwen_liu@sjtu.edu.cn/haiwen_liu@hotmail.com
} 
to improve the effective inductance. The proposed DGA is true 2-D structure which has vertical and horizontal periodicities of defected structure. Therefore, it has more degrees of freedom in terms of dimensions than the previous 1-D DGS. Measurements show that more excellent bandgap characteristics and higher slow-wave factors can be obtained from the proposed DGA than the existing periodic DGS in the same occupied surface.

\section{FRACTAL DEFECTED GROUND STRUCTURE AND ITS CHARACTERISTICS}

Figure 1 shows schematics of the proposed fractal DGS and a dumbbell DGS unit cell in the same occupied surface. The DGSs are etched on the metallic ground plane. The fractal

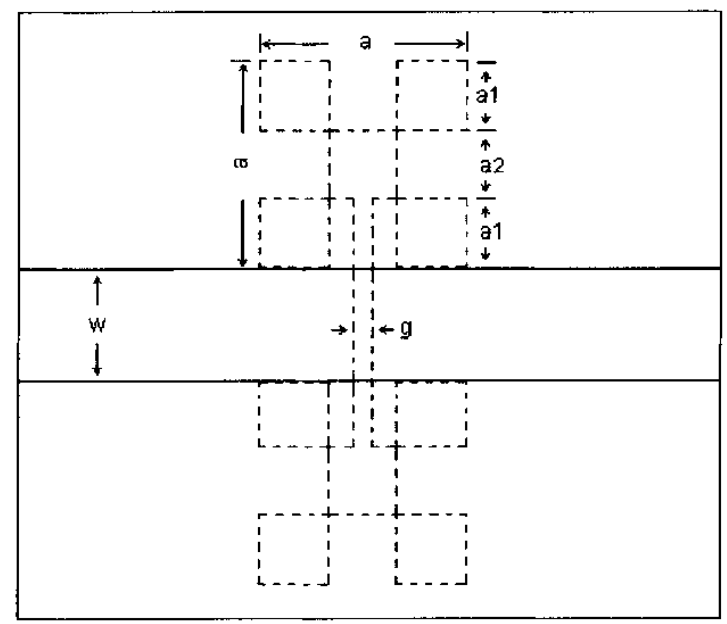

(a)

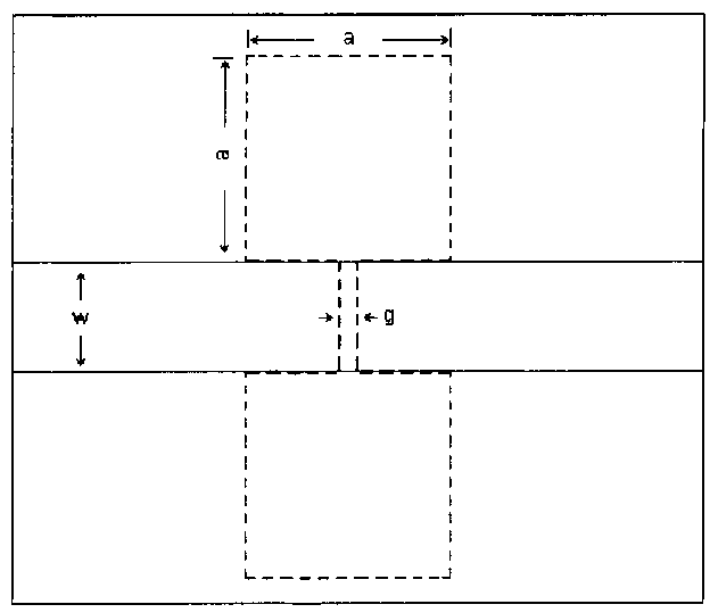

(b)

FIGURE 1 Schematic of microstrip line with DGS. $a=4.5 \mathrm{~mm}, w=2.4 \mathrm{~mm}$, and $g=0.4 \mathrm{~mm}$. (a) Fractal DGS $\left(a_{1}=1.8 \mathrm{~mm}\right.$ and $\left.a_{2}=0.9 \mathrm{~mm}\right)$; (b) dumbbell DGS. 


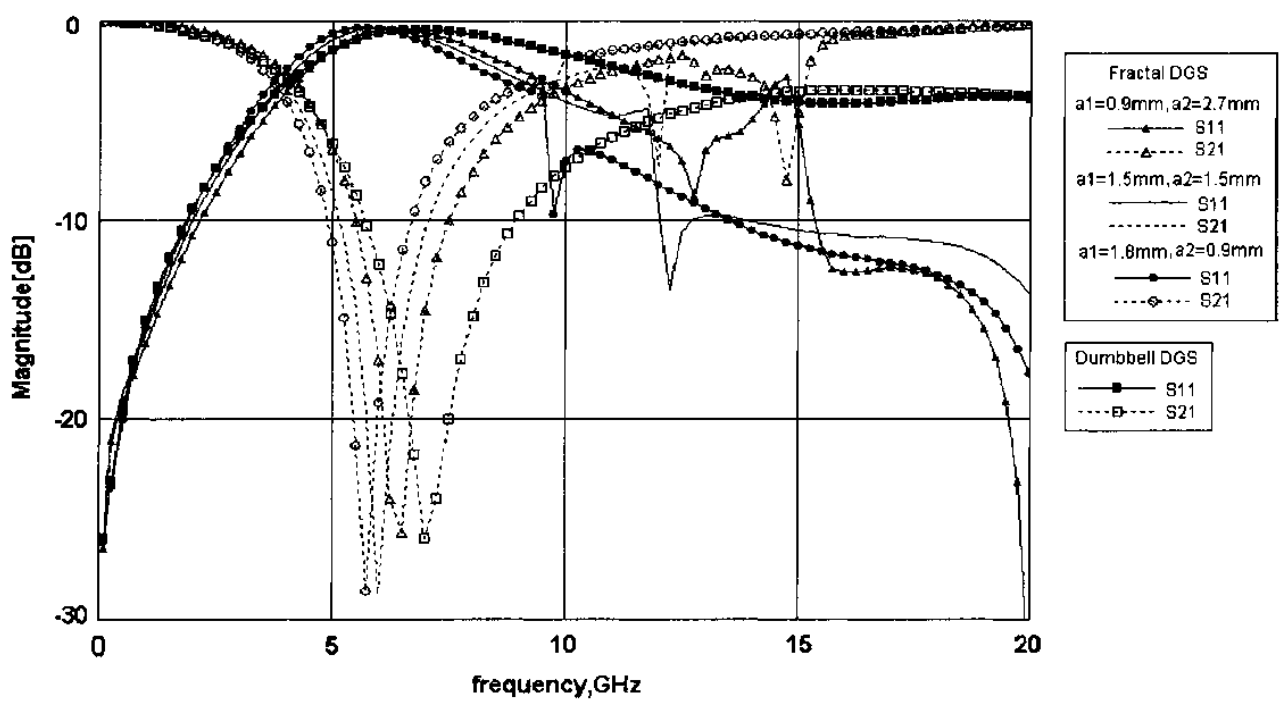

FIGURE 2 Electromagnetic simulation for the fractal and dumbbell DGS.

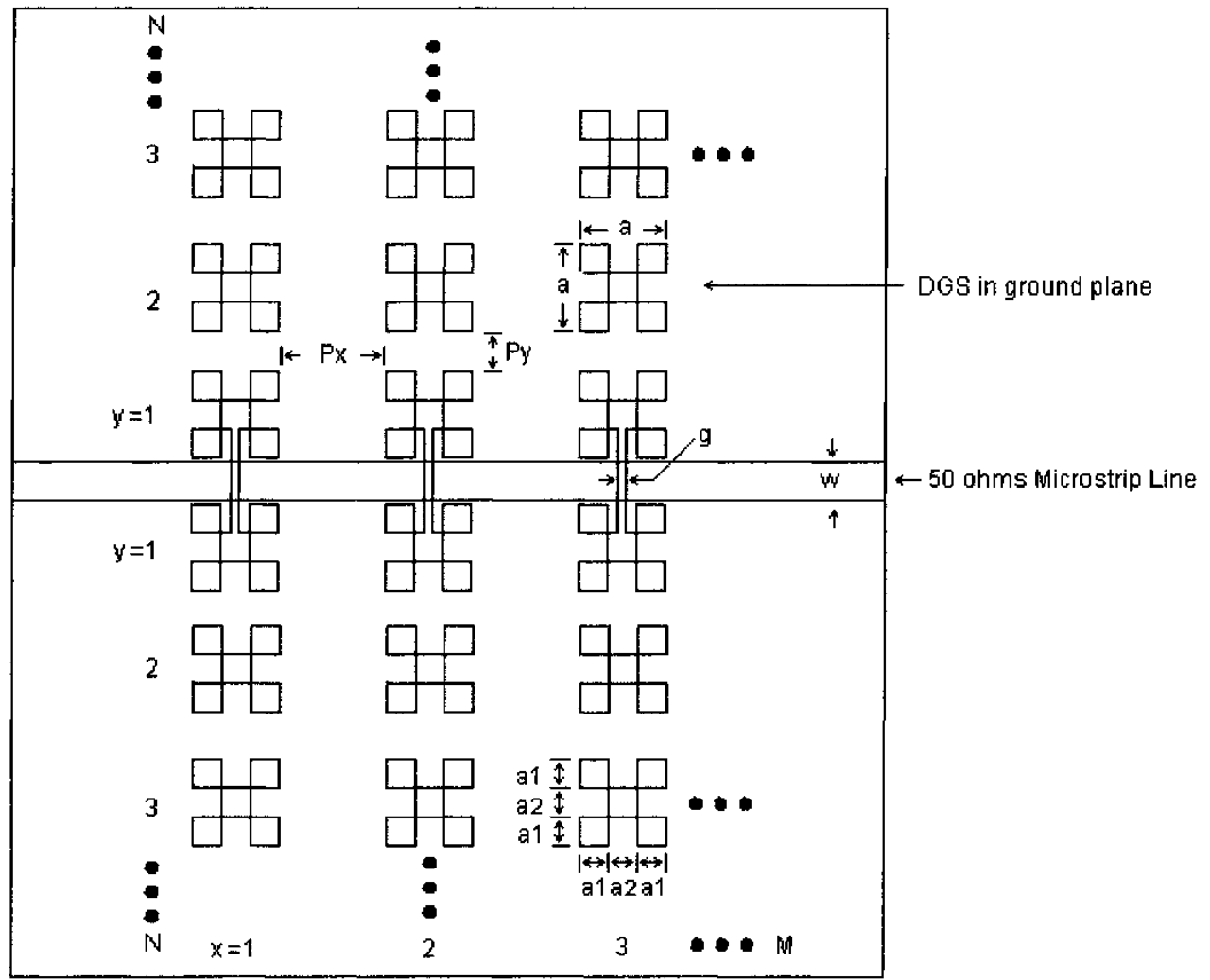

FIGURE 3 Microstrip line with DGA on the ground plane. 
defected unit cell possesses some self-similarity, just like a Sierpinski carpet, and shows some fractal characteristics [5]. The substrate for simulation is RT/Duroid 5880 with 31 mil of thickness and a dielectric constant $\varepsilon_{r}$ of 2.22. The line width $w$ is chosen for the characteristics impendance of $50 \Omega$ microstrip line.

Figure 2 shows the electromagnetic (EM) simulation of transmission characteristics of microstrip lines with DGS described in Figure 1. The simulation shows the attenuation poles of the fractal DGS shift lower than that of the dumbbell DGS in the same occupied

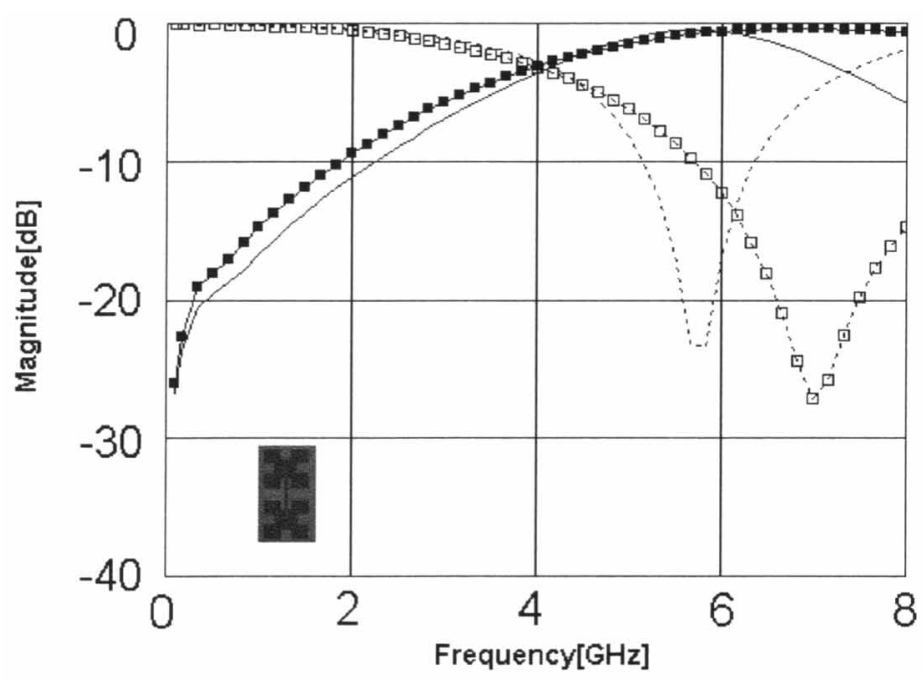

(a)

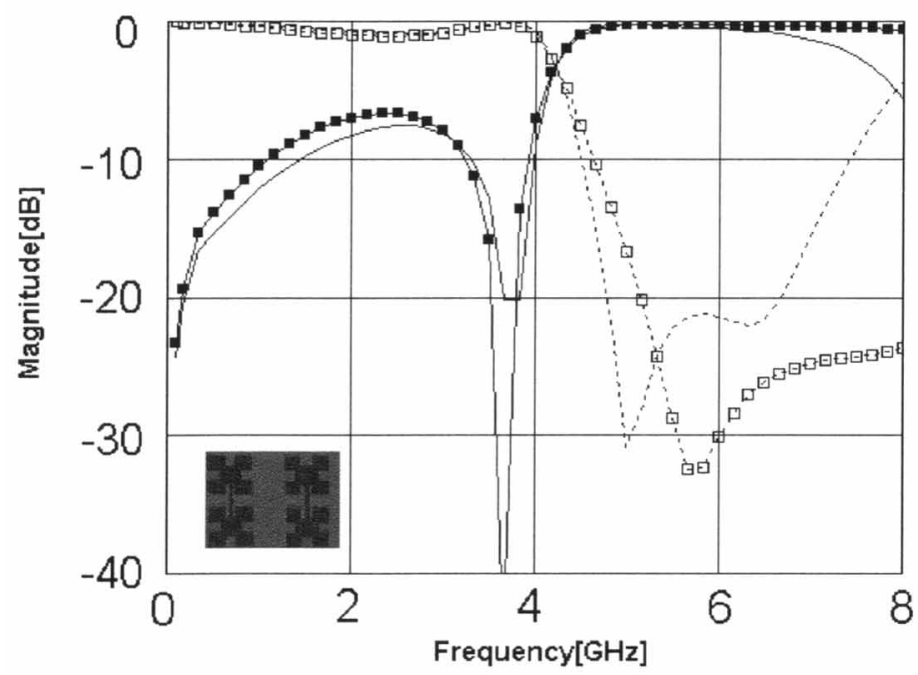

(b)

FIGURE 4 Measured characteristics of microstrip lines with various matrices of DGA. $(w=2.4 \mathrm{~mm}$, $g=0.4 \mathrm{~mm}, a=4.5 \mathrm{~mm}, a_{1}=1.8 \mathrm{~mm}, a_{2}=0.9 \mathrm{~mm}, P_{x}=3 \mathrm{~mm}$ and $P_{y}=1 \mathrm{~mm}$. The substrate is RT/Duroid 5880 with 31 mil of thickness and a dielectric constant $\varepsilon_{r}$ of 2.22.) (a) $x=1, y=1$; (b) $x=2, y=1$; (c) $x=2, y=2$; (d) $x=4, y=1$. 


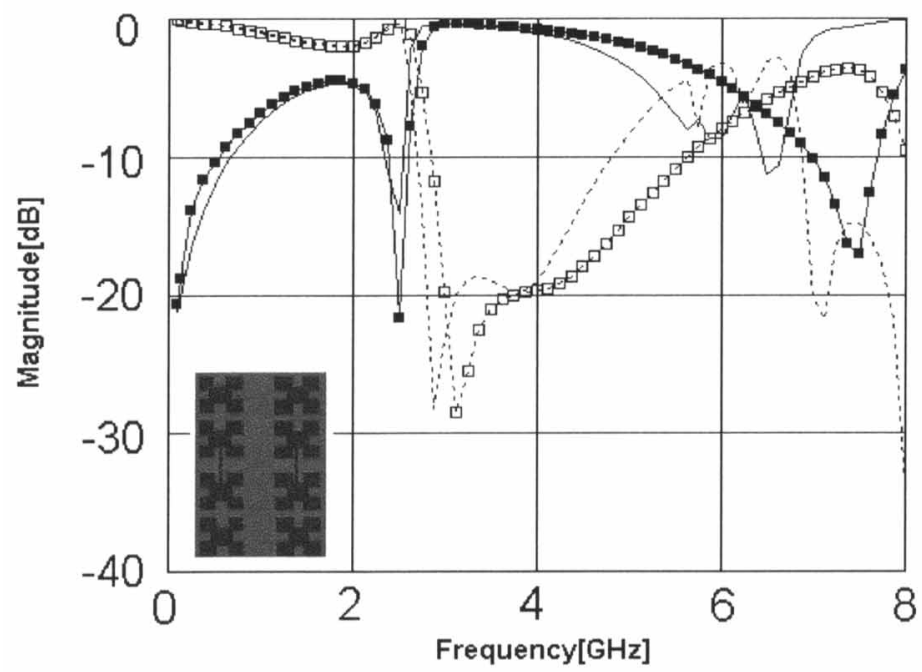

(c)

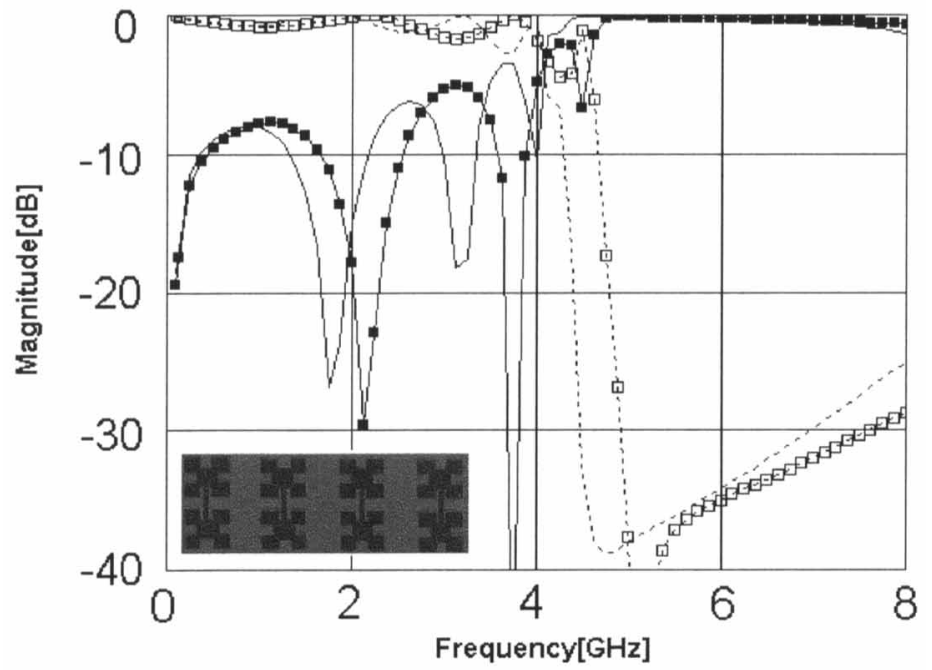

(d)

\section{S11(This work) -... S21(This work)}

$\longrightarrow-S 11(\operatorname{Ref}[9]) \cdots--S 21(\operatorname{Ref}[9])$

FIGURE 4 (Continued).

surface. Moreover, the slope of cutoff characteristics of the fractal DGS is steeper than that of the dumbbell DGS. So, the proposed fractal DGS has better bandgap characteristics. Additionally, the bandgap characteristics can be adjusted by the ratio of $a 1: a 2$. Electromagnetic simulation is using Sonnet 8.0. 


\section{STRUCTURE OF DEFECTED-GROUND ARRAY}

Figure 3 shows the structure of a microstrip line with DGA. The defect unit cell of DGA is composed of a Sierpinski carpet structure which has $a=2 a_{1}+a_{2} . x$ and $y$ mean the number of periodic defects along the horizontal and vertical direction, respectively, and are determined by the required frequency response. The defected unit cell of DGA can be expressed as $(x, y)=(1,1)$ if the position and number of defected unit cells are expressed as a matrix form for convenience. The horizontal and vertical periodicities of DGA are represented by $P_{x}$ and $P_{y}$, respectively. It is easy to obtain flexible frequency responses by adjusting the dimensions and vertical and horizontal periodicities.

\section{MEASURED RESULTS}

Figure 4 presents the measured characteristics of the proposed DGA, compared to the periodic DGS in Ref. [9]. Measurements show that DGA provides steeper cutoff characteristics and lower cutoff frequency. Also, the bottom views of the proposed DGA are shown in Figure 4. Furthermore, as the number of the defected unit cells is increased, the effective series inductance increases which gives rise to a much lower cutoff frequency, as seen in Figure 4. Figure 5 presents the comparison of the slow-wave factors of the proposed DGA and the dumbbell DGS for the same defected area on the ground plane. It is observed that the proposed DGA has higher slow-wave factors than dumbbell DGS.

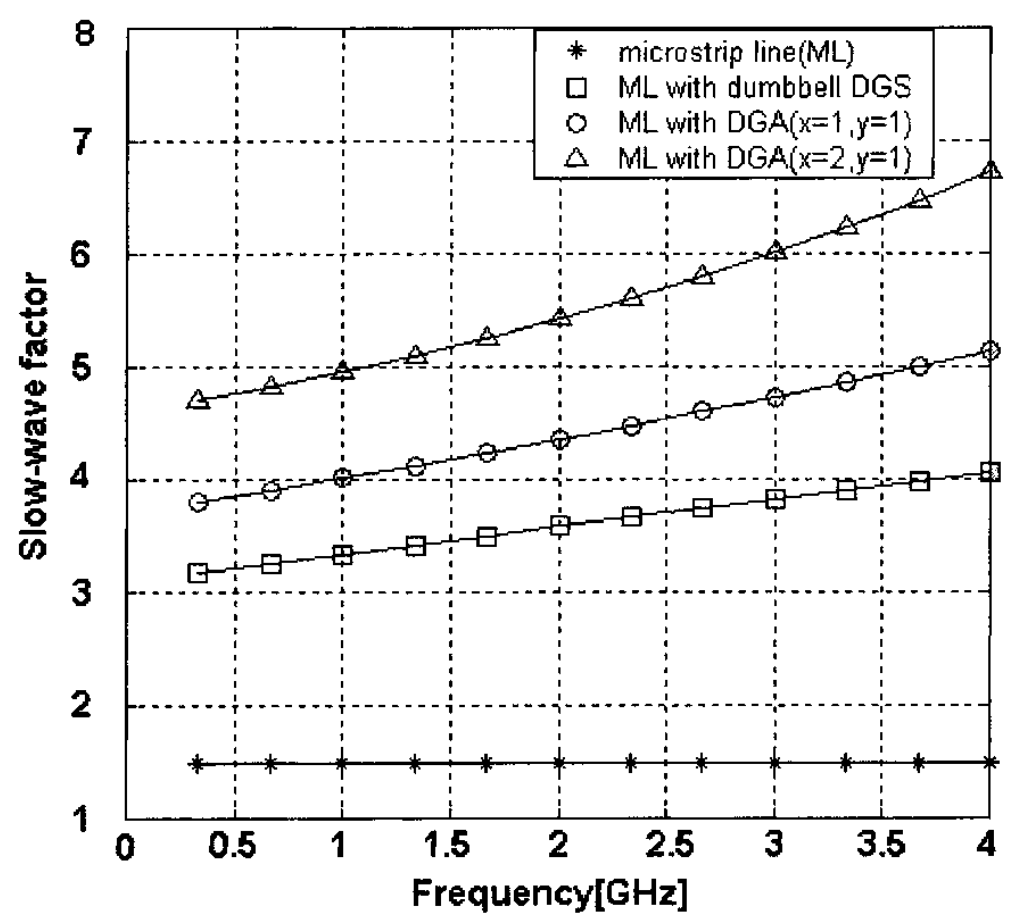

FIGURE 5 Comparison of the slow-wave factors. 
The above analysis shows that the proposed DGA can provide the size reduction of microstrip structure and bandgap characteristics more efficiently than the conventional periodic DGS.

\section{CONCLUSIONS}

A new 2-D DGA for planar circuits has been proposed in this paper, and has horizontal and vertical periodicities of defected structure. The defected unit cell of DGA is composed of a Sierpinski carpet structure to improve the effective inductance. Results show that the proposed DGA provides steep cutoff characteristics, low cutoff frequency, and high slowwave factors for planar circuits. It is expected that the potential applications will be extended widely to many RF and microwave circuits with MIC, MMIC, and LTCC technologies.

\section{References}

[1] Warns, C., Menzel, W. and Schumacher, H. (2001). Transmission lines and passive elements for multilayer coplanar circuits on silicon. IEEE Trans. Microwave Theory and Techniques, 49, 616-622.

[2] Caloz, C. and Itoh, T. (2002). Multilayer and Anisotropic Planar Compact PBG Structure for Microstrip Application. IEEE Trans. Microwave Theory and Techniques, 50, 2206-2208.

[3] Dal, A., Park, J. S., Kim, C. S., Kim, J., Qian, Y. X. and Itoh, T. (2001). A design of the low-pass filter using the novel microstrip defected ground structure. IEEE Trans. Trans. Microwave Theory and Techniques, 49, 86-93.

[4] Kim, C. S., Park, J. S., Dal, A. and Kim, J. (2000). A novel 1-D periodic defected ground structure for planar circuits. IEEE Microwave and Guided Wave Lett., 10, 131-133.

[5] Fu, Y. Q., Yuan, N. C. and Zhang, G. H. (2002). A Novel Fractal Microstrip PBG Structure. Microwave Optical and Tech. Lett., 12, 316-318.

[6] Lim, J. S., Park, J. S., Lee, Y. T., Dal, A. and Nam, S. (2002). Application of defected ground structure in reducing the size of amplifiers. IEEE Microwave and Guided Wave Lett., 12, 261-263.

[7] Lee, Y. T., Lim, J. K., Park, J. S., Dal, A. and Nam, S. (2002). A novel phase noise reduction technique in oscillator using defected ground structure. IEEE Microwave and Guided Wave Lett., 12, 39-41.

[8] Lim, J. S., Kim, C. S., Lee, Y. T., Dal, A. and Nam, S. (2002). A spiral-shaped defected ground structure for coplanar waveguide. IEEE Microwave and Guided Wave Lett., 12, 330-332.

[9] Lim, J. S., Kim, C. S., Lee, Y. T., Dal, A. and Nam, S. (2002). Vertically periodic defected ground structure for planar transmission lines. Electronics Lett., 38, 803-804. 

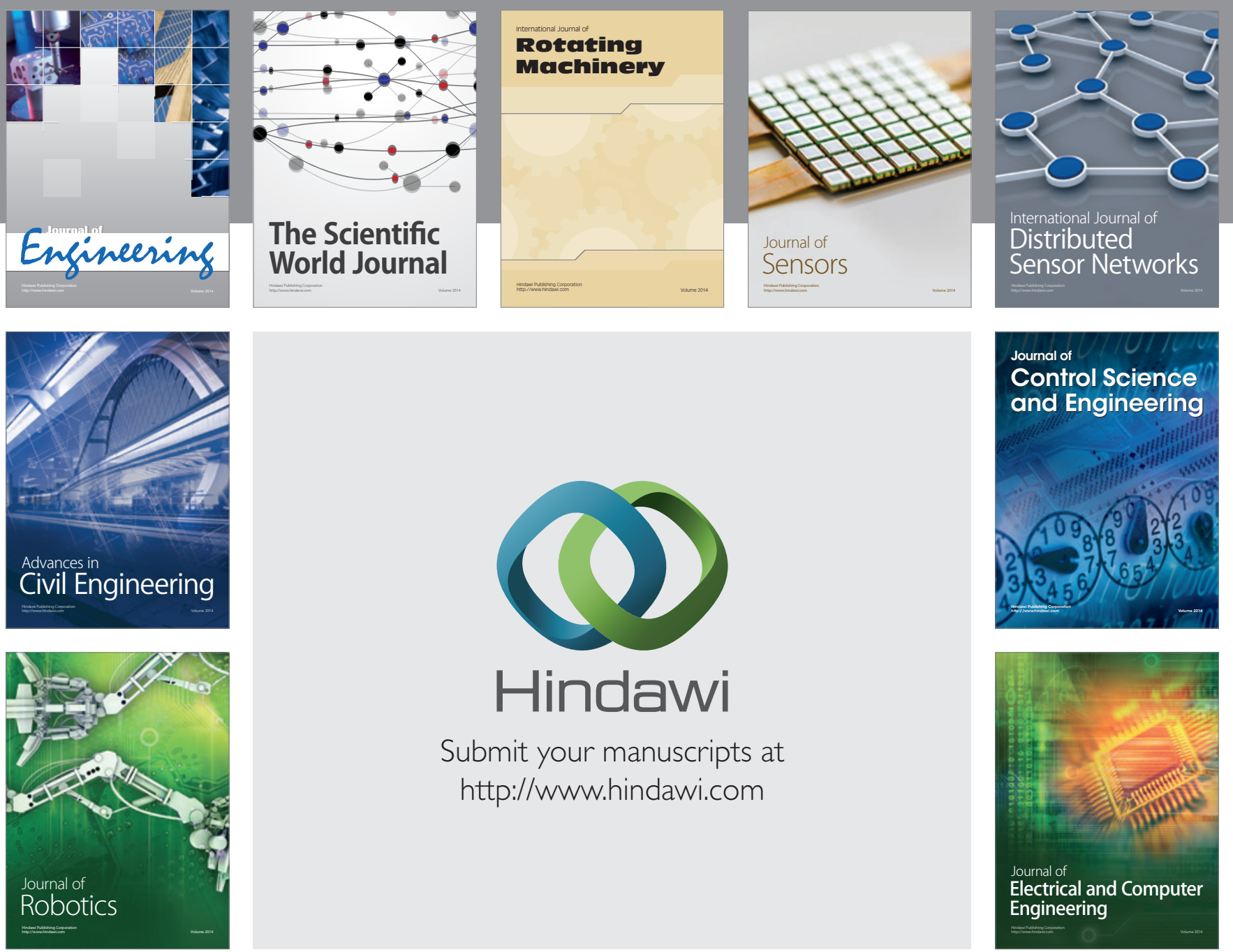

Submit your manuscripts at

http://www.hindawi.com
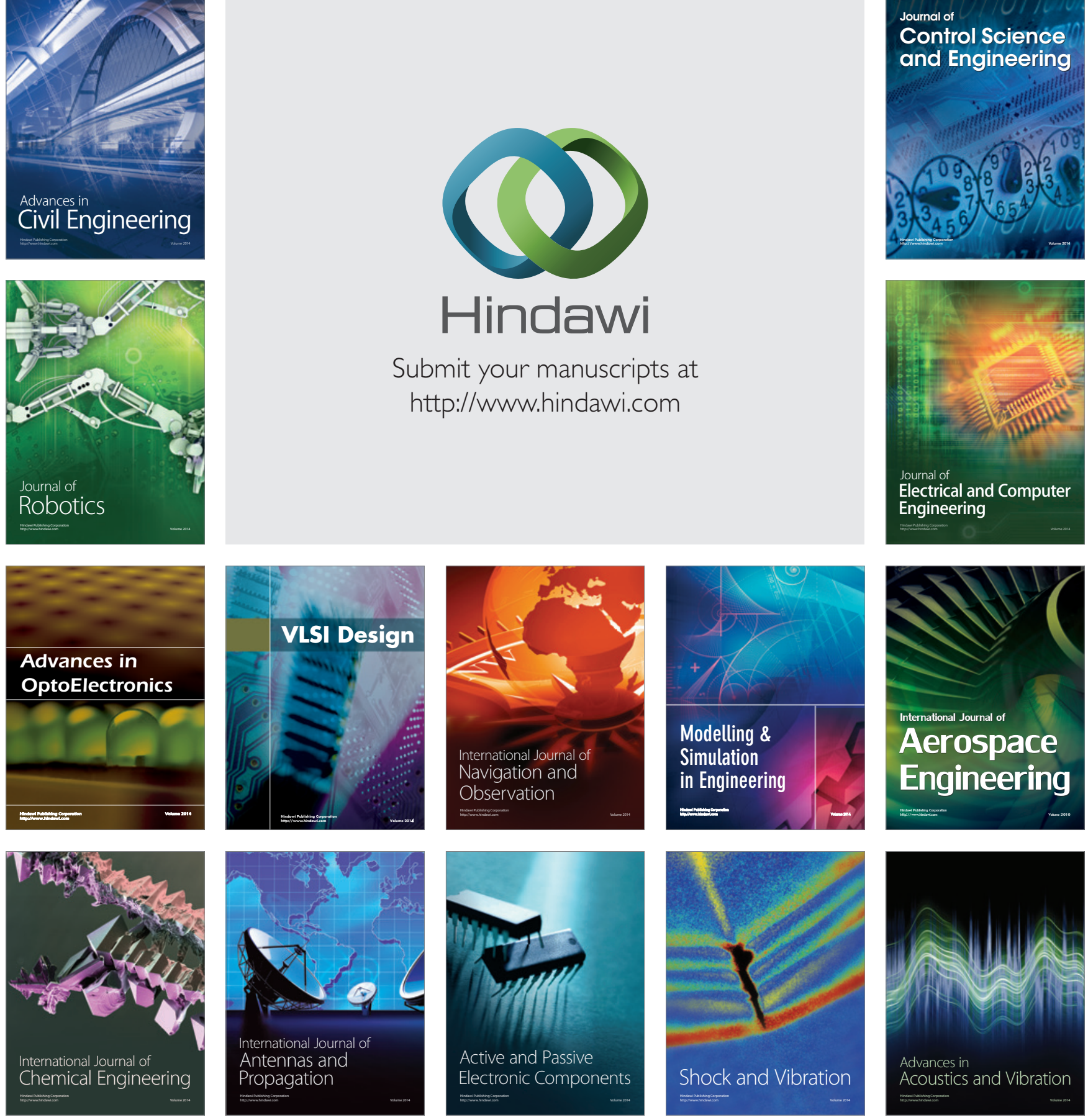